\title{
Studying the highly bent spectra of FR II-type radio galaxies with the KDA EXT model
}

\author{
Elżbieta Kuligowska
}

\author{
Astronomical Observatory of the Jagiellonian University, Orla 171 Cracow, Poland \\ e-mail: elzbieta@oa.uj.edu.pl
}

Received 9 July 2017 / Accepted 29 December 2017

\begin{abstract}
Context. The Kaiser, Dennett-Thorpe \& Alexander (KDA, 1997, MNRAS, 292, 723) EXT model, that is, the extension of the KDA model of Faranoff \& Riley (FR) II-type source evolution, is applied and confronted with the observational data for selected FR II-type radio sources with significantly aged radio spectra.

Aims. A sample of FR II-type radio galaxies with radio spectra strongly bent at their highest frequencies is used for testing the usefulness of the KDA EXT model.

Methods. The dynamical evolution of FR II-type sources predicted with the KDA EXT model is briefly presented and discussed. The results are then compared to the ones obtained with the classical KDA approach, assuming the source's continuous injection and selfsimilarity.

Results. The results and corresponding diagrams obtained for the eight sample sources indicate that the KDA EXT model predicts the observed radio spectra significantly better than the best spectral fit provided by the original KDA model.
\end{abstract}

Key words. galaxies: active - galaxies: evolution - galaxies: jets - radio continuum: galaxies

\section{Introduction}

The analytical models of FR II-type (Fanaroff \& Riley 1974) radio sources are based on the source's internal physics and characterise them in terms of dynamics, energetics, and luminosity evolution. These models originate from the "standard model" for double radio sources (Scheuer 1974; Blandford \& Rees 1974) in which FR II sources are composed of two opposite jets of plasma ejected from the vicinity of the supermassive black hole as a result of the accretion process. A travelling jet encounters the interstellar medium (ISM) of its host galaxy, the intergalactic medium (IGM), and finally the environment in the galaxy cluster, the intracluster medium (ICM). Its interaction with those media results in the formation of the terminal bow shock and the interface between the shocked and unperturbed external material and the relativistic electrons reaccelerated in the so-called hot spot regions.

The more sophisticated models for FR II-type sources such as KDA (Kaiser et al. 1997), BRW (Blundell et al. 1999) and MK (Manolakou \& Kirk 2002) differ from the standard model in the assumptions on the transport mechanisms of the new particles between the jets, hot spots, and lobes. While the KDA model assumes a constant injection index for the particles, in the BRW model this index varies between the different energy regimes. However, the MK model again assumes a constant injection index, but also takes into account the reacceleration of the particles in the turbulent processes occurring during their transport to the lobe. It is worth adding that Barai \& Wiita (2006) performed detailed quantitative tests of three popular models describing the evolution of FR II radio sources (KDA, BRW and MK) based on multidimensional Monte Carlo simulations. Their main aim was to compare the predictions of these models with the observational data (samples constructed based on low-frequency radio surveys revised Third Cambridge Catalogue of Radio Sources (3CRR, Laing et al. 1983), revised 6C (6CE, Rawlings et al. 2001) and 7C Redshift Survey (7CRS, Willot et al. 2001). Barai and Wiita concluded that none of the examined models produce fully satisfactory results (acceptable fits to all the properties of the samples). However, their statistical test suggests that the KDA model still appears to provide better results than the Manolakou \& Kirk and Blundell et al. models.

The KDAEXT model originally published by Kuligowska (2017) arose from the need to better understand the processes occurring in FR II-type radio sources after the end of their nuclear activity. In contrast to the previously developed models describing the dynamics of these sources and based on the "standard model", such as KDA, BRW and MK, KDA EXT was constructed on the assumption of the lack of so-called continuous injection $(\mathrm{CI})$ of new particles into the lobes at the time at which the given source is observed. This implies that the radio lobes are no longer powered by the central active galactic nucleus (AGN), and the whole source's structure begins to dissipate at radio wavelengths.

Non-thermal continuum radio emission of the lobes is due to the synchrotron process and to the inverse-Compton scattering of ambient photons of the cosmic microwave background. The synchrotron radiation results from the ultra-relativistic particles interacting with the magnetic field, with the most energetic ones emitting energy at high radio frequencies and losing it at the fastest rate. Thus in the case of sources with no injection of new particles from their nucleus, one should be able to observe a substantial steepening of their spectra. In fact such FR II-type sources, with radio spectra highly bent at $\mathrm{GHz}$ frequencies, are known. Among them are both very weak radio relics (Shulevski et al. 2015) and objects with so-called recurrent activity (Saikia et al. 2010), as well as the more typical, powerful radio galaxies 
with curved spectra (i.e., the ones modeled and discussed by Kuligowska (2017). It is worth emphasising that the problem of evolution of FR II-type sources after their jet activity has stopped was previously widely discussed by Kaiser \& Cotter (2002). They argue that, after the jet switches off, the information about ceasing of the lobes should propagate with the speed of sound. If the internal sound speed in the lobes is relatively low, the adiabatic evolution of the cocoon is unchanged for a long period of time. Lobes may still be overpressured with respect to the ambient gas and continue their expansion (model A). However, if the sound speed is fast, the entire radio structure almost immediately transforms the so-called coasting phase and its adiabatic evolution slows down rapidly (model B).

In the present paper, the suitability of the KDAEXT model for studying the FR II-type sources with bent spectra is further analysed. However, instead of finding examples of such sources in a wide range of available literature or radio catalogues, the present study is focused on the large and representative sample of 388 bright radio sources observed at $74 \mathrm{MHz}$ (Helmboldt et al. 2008). This sample was used for the several reasons: It is big enough to ensure the presence of sources with various spectral shapes; it consists of sources that are powerful at low radio frequencies, allowing reliable modelling of their dynamics with KDA or KDA-like models; and it provides ready-to-use flux densities of the sources, available over a wide range of the radio spectrum. Here, the analytical formulae for the dynamics and luminosity evolution of the lobes, given by the KDA EXT model, is applied to the eight FR II-type sources with presumably terminated jet inflow carefully selected from the Helmboldt et al. (2008) sample.

The organisation of this article is as follows: the summary of the KDA EXT model and its comparison with the original KDA model is provided in Sect. 2. The description of the sample of Helmboldt et al. (2008) is given in Sect. 3 along with the methodology for further selecting the sources with strongly curved spectra, for which the available observations are sufficient to obtain satisfactory and accurate models. Section 4 presents the method of fitting both the KDA EXT and KDA models to the observational data of the selected radio galaxies, as well as the results (radio spectra obtained with the models). Finally, Sect. 5 presents a brief summary of these results.

\section{The summary of KDA and KDA EXT models}

\subsection{KDA and its background}

The KDA model is based on a dynamical description developed by Kaiser \& Alexander (1997) and further combined with the radiative processes analysed by Kaiser et al. (1997). Its most important assumptions are: power-law radial density distribution (King's 1972 model) of unperturbed ambient gas surrounding the radio source, continuous injection of new particles into the jet, and energy conservation within the jet. The model predicts (among others) the most evident observational parameters of a radio source, that is, the total length of its jets (identified with lobes) and its total radio power at a given frequency. The final equations for these parameters are given below, and their derivation is presented by Kaiser et al. (1997).

The length of the jet at a given source's age is given by

$r_{\mathrm{j}}(t)=c_{1}\left(\frac{Q_{\mathrm{j}}}{\rho_{0} a_{0}^{\beta}}\right)^{1 /(5-\beta)} t^{3 /(5-\beta)}$, where $\beta$ is the exponent of the density profile, $a_{0}$ is the size of the radio core, $\rho_{0}$ is the central density of this core, $Q_{\mathrm{j}}$ is the jet's power and $r_{\mathrm{j}}$ is identified with one-half of the source's linear size $D, r_{\mathrm{j}}=D / 2$. If two of the model parameters, $Q_{\mathrm{j}}$ and $\rho_{0} a_{0}^{\beta}$, are specified, the model predicts the time evolution of the source's length.

The radio emission of the source is calculated by splitting the cocoon surrounding the jet into infinitesimal evolving volume elements and tracking its total adiabatic and radiative losses. The sum of the contributions from volume elements gives the total emission (at a frequency $v$ ), $P_{v}(t)$, as a integral over the injection time $t_{\mathrm{i}}$, which can then be solved numerically.

$$
\begin{aligned}
P_{v}(t)= & \int_{t_{\min }}^{t} \mathrm{~d} t_{\mathrm{i}} \frac{\sigma_{T} c r}{6 \pi v(r+1)} Q_{\mathrm{j}} n_{0}\left(P_{\mathrm{hc}}\right)^{\left(1-\Gamma_{\mathrm{c}}\right) / \Gamma_{\mathrm{c}}} \\
& \times \frac{\gamma^{3-p_{i}} t_{\mathrm{i}}^{a_{1} / 3(p-2)}}{\left.\left[t^{-a_{1} / 3}-a_{2}\left(t, t_{\mathrm{i}}\right) \gamma\right)\right]^{2-p}}\left(\frac{t}{t_{\mathrm{i}}}\right)^{-a_{1}\left(1 / 3+\Gamma_{\mathrm{B}}\right)},
\end{aligned}
$$

where $\left(P_{\mathrm{hc}}\right)$, the ratio of the jet head pressure $\left(p_{\mathrm{h}}\right)$ and the uniform cocoon pressure $\left(p_{\mathrm{c}}\right)$, is a function of $\left(R_{\mathrm{T}}\right)$ - the axial ratio of the cocoon described by the empirical formula adopted from Kaiser (2000), $\Gamma_{c}$ and $\Gamma_{B}$ are the adiabatic indices in the equation of state of the cocoon material and the magnetic field, respectively, $\sigma_{T}$ is the Thompson cross-section, $c$ is the speed of light, $r$ is the ratio of the energy density of the magnetic field $u_{B}, a_{1}$, and $a_{2}$ are the physical constants defined by Kaiser et al. (1997), $n_{0}$ is the number density of electrons (Heavens \& Drury 1988), $p$ is the exponent of the energy distribution of jet particles, and $\gamma$ is their Lorentz factor.

\subsection{KDAEXT model}

The idea of developing the KDAEXT model grew out of the need for an estimation of the age of the source at which its nuclear activity starts to cease (hereafter referred as $t_{\mathrm{br}}$ ). One can assume that stopping this activity ceases the inflow of new particles into the jet and eventually reduces the radio brightness of the entire radio structure. For such sources, the KDA model may not be reliable (even if it can formally be fitted to the observed data), because its basic condition of a continuous injection process is no longer fulfilled. The extended model should then consider both the new parameter describing the source's dynamics $\left(t_{\mathrm{br}}\right)$ and the overall evolution of the source after the jet stops.

The KDAEXT model (Kuligowska 2017) resulting from these theoretical considerations allows us to predict the lobe (jet) length and radio power of the source at a given frequency. It is assumed that the termination of the energy supply propagates from the AGN to the lobes with a relatively low sound speed (Kaiser et al. 2000) and, after switching off the jets, the adiabatic evolution of the lobes of the large sources does not change significantly for a long time. In particular, the lobes may still be overpressured with respect to the external gaseous environment and therefore continue to expand behind a bow shock, as described by Kaiser \& Cotter (2002). In the paper of Kuligowska (2017), the effective formula for lobe length, describing its adiabatic evolution after termination of the activity and compared to the analogous equation given by the KDA model, is based on the work of Kaiser \& Cotter and derived as:

$D\left(t, t_{\mathrm{br}}\right)=\left\{\begin{array}{ll}c_{1}\left(\frac{Q_{\mathrm{j}}}{\rho_{0} a_{0}^{\beta}}\right)^{1 /(5-\beta)} t^{3 /(5-\beta)} & \text { for } t<t_{\mathrm{br}} \\ D\left(t_{\mathrm{br}}\right)\left(\frac{t}{t_{\mathrm{br}}}\right)^{\frac{2\left(\Gamma_{\mathrm{c}}+1\right)}{\Gamma_{\mathrm{c}}\left(7+3 \Gamma_{\mathrm{c}}-2 \beta\right)}} & \text { for } t \geq t_{\mathrm{br}}\end{array}\right.$, 
where $D\left(t_{\mathrm{br}}\right)$ is the cocoon length at the time of switching off the energy supply. Hereafter the Kaiser \& Cotter's approach in the limiting case when the internal sound speed is fast (their model B) is adopted.

The energy-loss process due to the synchrotron and inverseCompton scattering of the Cosmic Microwave Background (CMB) photons is characterised by the energy break in the energy spectrum comprised of particles with different energies. In the case of a source supplied by a constant flow of particles, the highest break frequency (for the pitch angle $\theta=90^{\circ}$ ) is given by:

$v_{\mathrm{br}, \mathrm{CI}}=C_{1} B \gamma_{\mathrm{br}}^{2}=\frac{C_{1}}{C_{2}^{2}} \frac{B}{\left(B^{2}+B_{\mathrm{iC}}^{2}\right)^{2} t^{2}}$,

which is assumed to be valid for $t \leq t_{\mathrm{br}}$, where $B$ and $B_{\mathrm{iC}}$ are the magnetic field strengths related to the synchrotron and inverse-Compton losses, and $C_{1}$ and $C_{2}$ are the physical constants defined by Pacholczyk (1970). However, if the pitch angle of the particle distribution is highly isotropic (Jaffe \& Perola 1973), the break frequency valid for $t>t_{\mathrm{br}}$ is given by:

$v_{\mathrm{br}, \mathrm{JP}}=\frac{C_{1}}{C_{2}^{2}} \frac{B}{\left\{\left(2 / 3 B^{2}+B_{\mathrm{iC}}^{2}\right)\left(t-t_{\mathrm{br}}\right)\right\}^{2}}$,

where $C_{1} / C_{2}^{2}=2.51422 \times 10^{12}$ and $B_{\mathrm{iC}}=0.318(1+z)^{2} \mathrm{nT}$.

Thus the analytical formula for the total radio power of a source at a given frequency is the simple sum of these two integrals over time $t$. In the context of the KDA EXT model, the first of them is the total source power until the time of the jet switchoff, and the second one represents its radio power emitted from $t_{\mathrm{br}}$ until the actual age of the observed source, $t$. The total power of a source is given by:

$P_{v}(t)=\left\{\begin{array}{ll}P_{v}\left(t_{\mathrm{min}}, t_{\mathrm{br}}\right)+P_{v}\left(t_{\mathrm{br}}, t\right) & \text { for } t_{\mathrm{br}}>t_{\mathrm{min}} \\ P_{v}\left(t_{\mathrm{min}}, t\right) & \text { for } t_{\mathrm{br}} \leq t_{\mathrm{min}}\end{array}\right.$,

where the first top term is the integral given by Eq. (2) with the limit of integration $t_{\min }$ changed to $t_{\mathrm{br}},\left(t_{\mathrm{min}}\right.$, defined by Kaiser et al. (1997), is the minimum injection time for which the lobe material is still radiating at a given frequency $v$ ), and the second top term corresponds to Eq. (9) of Kuligowska (2017). The lower integral refers to the classical KDA integration given by Eq. (2).

\section{Selected sample of FR II-type sources with aged radio spectra}

\subsection{Helmboldt's sample of 388 bright radio sources at $74 \mathrm{MHz}$}

The KDAEXT model is expected to predict observational parameters (i.e., jet length, radio power value at given frequency) of those FR II-type radio sources in which nuclear activity is terminated or suspended. It is assumed that the continuum injection KDA model does not provide accurate fits of their observed radio spectra. While most of the known sources are active and can be fit with the KDA model, KDA EXT is also able to reproduce the curved spectra of the dying radio sources. However, it should be emphasised that KDA EXT model is still useful in the study of active sources with the CI process: it represents a generalisation of the classical KDA model, and it can be easily reduced to the KDA model by setting one of its free parameters $\left(t_{\mathrm{br}}\right)$ to zero.
One can assume that jet termination usually entails a rapid decrease of the source's radio flux density which is most significant at the highest frequencies (depending on the value of $t_{\mathrm{br}}$ : from about $5 \mathrm{GHz}$ and up to $20-50 \mathrm{GHz}$ or even more) - as previously mentioned in Sect. 1. In this work we select sources with extremely bent spectra rather than simply the sources with very steep radio spectra.

In the previous paper of Kuligowska (2017) six examples of such sources were presented. This previous "test" sample for the KDA EXT model was compiled from literature and composed of a variety of radio galaxies, including giant radio galaxies (GRGs) and radio sources with restarting activity. Although the final sample included some relatively young objects, it was constructed with the key assumption that predominantly old and rather large sources should have their radio spectra significantly bent towards the highest frequencies. Here this presupposition is abandoned. It is rather simply assumed that any sources with bent spectra may be studied with the KDA EXT model, regardless of their other observational parameters, such as linear size, age, or redshift. The additional condition to be met by such sources is their relatively high radio brightness - in particular, the high value of radio flux density at low frequencies, which gives a better chance of good spectrum coverage over a wide range of radio frequencies (cf. Sect. 3.3).

For the above reasons, the sample of 388 radio sources compiled by Helmboldt et al. (2008) should be perfect in the search for those sources. First, it seems to be big enough to provide a representative subsample of objects of interest. Second, it was compiled from the available literature for all sources within the VLA Low Frequency Sky Survey (VLSS) brighter than $15 \mathrm{Jy}$ at $74 \mathrm{MHz}$, providing only the most powerful radio sources. Moreover, the sample also contains carefully collected radio frequency spectra for these sources, provided in the literature and compiled using the corrections needed to put the flux densities from all the references on the same absolute flux density scale of Baars et al. (1977). It follows that the sample of Helmboldt gives a convenient list allowing research on the ready-made radio spectra of these sources. It is also worth noting that the flux densities measured by VLSS are more reliable than many others from older low-frequency surveys, and the sky coverage of VLSS itself is large enough to provide a satisfactory spatial diversity of the sample sources.

\subsection{Selection criteria}

As mentioned above, the primary criterion is the strong collapse of the source's radio spectrum at the highest frequencies. To extract such sources, firstly all the source spectra provided by Helmboldt were visually inspected, and all objects without highly bent spectra, typical for sources with continuous activity, were rejected, as well as those which were evidently not FR II-type radio sources, due to previous classification or their different spectral shapes (i.e., compact steep spectrum (CSS) sources with spectra peaking around $100 \mathrm{MHz}$ or lower, or $\mathrm{GHz}$ peaked spectrum (GPS) sources with inverted spectra). After this initial step, 47 previously selected radio sources were carefully examined for the completeness of the observational data required for the application of the KDA EXT model. In particular, this model needs broad (wide enough to include very low and high frequencies) coverage of the radio spectrum (this criterion was valid for all cases) and a redshift value determined for the parent radio galaxy (unknown for almost one third of the initially selected objects). However, the most important was the availability of the radio maps. They are not only necessary to correctly 
determine the length and thickness of the lobes (input parameters for both KDA EXT and KDA models), but also to confirm the FR II-type morphology of the given source, provided by the literature or online data catalogues. In particular, radio maps usually clearly show the presence of bright radio lobes and hot spots. Unfortunately, good-quality radio maps are not available in the case of 15 sources of the preliminary sample. Moreover, despite the shapes of their radio spectra, the maps of two previously selected sources (3C048 and NGC1692) do not show the characteristic FR II morphology. After further verification, 3C048 turns out to be an example of a core-dominated source (Laing et al. 1983), while NGC1692 (0453-20) was classified as a source with "diffuse and amorphous radio emission" by Morganti et al. (1993). The radio galaxy 3C217 fulfils all the selection criteria, however it was previously examined with the same KDA EXT model in the earlier paper by Kuligowska (2017). For this reason its KDA EXT analysis is not repeated in this work, and the reader is referred to that publication.

In the next step, the progression of the radio spectrum slope for the remaining 26 sources was further investigated. The spectral index $\alpha_{v}$, changing along with the spectrum, was measured and its slope gradient was calculated for every pair of neighbouring radio flux densities. Here the author assumes that the so-called high curvature (bend) of the given spectrum must result in: (1) high $\left(\alpha_{v} \sim 1.5\right)$ spectral indices occurring in the highest-frequency part of the spectrum, implying a highfrequency slope significantly exceeding the limiting value of $\alpha_{\text {inj }}+0.5$ expected in the CI process; (2) violent steepening of the spectrum between the frequencies in the range of $1400 \mathrm{MHz}$ up to $4800,10000 \mathrm{MHz}$ or even $20000 \mathrm{MHz}$, giving an increment of $\alpha_{v}$ of nearly one between these data points; and (3) relatively low spectral index (not exceeding 0.6) for the low-frequency flux densities (26-178 MHz).

The above considerations finally led to the further limitation of the initial sample to nine sources: 3C006.1, 3C032, 3C041, 3C123, 3C247, 3C300, 3C325, 3C401, and PKS 0235-19. Further, $3 \mathrm{C} 300$ was excluded because its radio spectrum is very irregular and does not allow for a reliable fit of the KDA EXT model due to its repeated breaks and flattening at higher frequencies. The final sample includes almost exclusively $3 \mathrm{C}$ sources and consists of objects of rather mid-scale linear sizes $(68-386 \mathrm{kpc})$ and relatively low ( $z$ up to 0.84$)$ redshifts, except $3 \mathrm{C} 325$ whose high $(z=1.135)$ redshift makes this source especially interesting from the viewpoint of studying the processes of interruption of AGN activity.

In summary, the final number of dying FR II-type sources selected from Helmboldt's compilation and fulfilling the formal criteria (specific steepening of their spectra) is of the order of 21 , which is $5.4 \%$ of the initial sample. However, only 8 of these are further investigated here for the reasons given above (lack of sufficient data, irregular spectrum). At this point it would be interesting to provide the percentage of all the sources with terminated jet activity drawn from the complete population of FR II- and FR I-type sources. Unfortunately, this is rather problematic due to the difficulty in estimating the total number of such radio sources as well as detecting the weak radio emission of dying radio galaxies. However, Giovannini et al. (1988) argue that only a few percent of all radio sources are dying, based on data from Bologna (B2, Colla et al. 1970) and Third Cambridge Catalogue of Radio Sources (3C, Edge et al. 1959) catalogues. This result is in line with the percentage obtained for Helmboldt's sample. It is also worth emphasising (Murgia et al. 2011) that dying radio sources are not synonymous with the radio relics, typically not associated with individual galaxies.

\subsection{The summary of observational data for the sample sources}

Table 1 presents the observational data of the sample sources used for testing the usefulness of the KDAEXT model. Redshifts are given with the highest known accuracy available in the literature. Despite the undefined values of some redshift uncertainties, it is assumed that the errors of the given spectroscopic redshifts are very small and do not affect the result of modelling. The 'largest angular sizes' ( $L A S)$ and $R_{t}$ values of axial sources are estimated based on the accessible radio maps (along with the uncertainties given by their angular resolution). The projected linear sizes of the sources, $D$, are calculated following the formula $D=4.848 \times 10^{-6} L_{\mathrm{A}}(z) L A S$ and their measurement uncertainties are calculated with the partial derivatives. Another model parameter, inclination angle of the jet axis $\theta$, is not listed in the table because in the case of all the analysed sources it can be assumed to be close to $90^{\circ}$ (sources are not significantly inclined to the line of sight). Moreover, it can be demonstrated that a slight deviation of this angle does not significantly affect the modelling results.

Table 1 also includes the flux density values of the sources at $74 \mathrm{MHz}$. The remaining flux densities for a large set of frequencies and their errors are derived from Helmboldt et al. (2008). It is worth noting here that the original uncertainties on the measurements are often underestimated (further discussed in the following Section). Due to the large number and variety of the available flux densities, these data are not listed in the table, but are provided in the individual source summaries (Sect. 4).

Before fitting the model parameters (cf. following Section), all the flux densities derived following Helmboldt were inspected and some clearly undervalued data points (i.e. high frequency measurements covering the hot spots only) were removed from the input sets for the certain sources. Additionally, the $150 \mathrm{MHz}$ TIFR GMRT Sky Survey (TGSS, Intema et al. 2017) flux measurements available for all eight sources were added and used in the modelling.

The values of the sources' radio power $P_{v}$ are calculated from the given flux densities according to the formula $P_{v}=S_{v} L_{\mathrm{D}}(z)^{2}(1+z)^{\left(\alpha_{v}-1\right)}$, where $L_{\mathrm{D}}(z)$ is the luminosity distance of the given source determined assuming a flat Universe with Hubble constant $H_{0}=71 \mathrm{~km} \mathrm{~s}^{-1} \mathrm{Mpc}^{-1}$ and the $\Lambda$ cold dark matter $(\Lambda C D M)$ model with cosmological $\Omega_{\mathrm{m}}=0.27$ and $\Omega_{\Lambda}=0.73$, and where $\alpha_{v}$ is the spectral index measured as the spectrum slope gradient calculated separately for every pair of neighbouring radio flux densities. Moreover, determining the initial dynamical parameters for both the KDA and KDA EXT models requires at least five sources' radio power $P_{v}$ values at significantly distant observing frequencies for the reliable application of the models.

We include recent $150 \mathrm{MHz}$ TGSS flux densities to increase the number of measurements and accuracy of the calculations. However, for the sources 3C41, 3C123, and PKS 0235-19 the $150 \mathrm{MHz}$ data points clearly stand out from the trend lines. In these cases, the use of the TGSS flux densities in the modelling does not improve the accuracy of the fits, and the use of these values is debatable. Moreover, the TGSS flux densities of some sources are recognised as having systematically underestimated values (up to even 50\%). Special fixes to the original TGSS, resulting in the revised Rescaled Subset of the Alternative Data Release 1 (TGSS-RSADR1) catalogue, were recently proposed by Hurley-Walker (2017). Thus TGSS flux densities for these three sources have been verified by cross checking bright point-like sources in their vicinity (comparing their TGSS flux 
E. Kuligowska: Studying the highly bent spectra of FRII-type radio galaxies with the KDAEXT model

Table 1. Observational data for FR II-type sources selected from the sample of Helmboldt et al. (2008).

\begin{tabular}{lllllllll}
\hline \hline Name & $3 \mathrm{C} 006.1$ & $3 \mathrm{C} 32$ & $3 \mathrm{C} 41$ & $3 \mathrm{C} 123$ & $3 \mathrm{C} 247$ & $3 \mathrm{C} 325$ & 3C401 & PKS 0235-19 \\
\hline$z$ & $0.840 \pm 0.0004$ & 0.400 & $0.795 \pm 0.001$ & $0.2177 \pm 0.0003$ & 0.7489 & 1.135 & 0.201 & 0.620 \\
$\left.L A S{ }^{\prime \prime}\right]$ & $32 \pm 3$ & $72 \pm 1$ & $26 \pm 1$ & $41 \pm 3$ & $25 \pm 3$ & $29 \pm 4$ & $24 \pm 2$ & $45 \pm 2$ \\
$R_{t}$ & $3.3 \pm 2.2$ & $4.6 \pm 0.6$ & $4.6 \pm 1.6$ & $4.0 \pm 1.5$ & $1.5 \pm 0.7$ & $1.7 \pm 1.0$ & $3.0 \pm 1.7$ & $1.5 \pm 0.27$ \\
$D[\mathrm{kpc}]$ & $246 \pm 23$ & $386 \pm 5$ & $200 \pm 8$ & $123 \pm 11$ & $188 \pm 22$ & $235 \pm 33$ & $68 \pm 7$ & $310 \pm 14$ \\
$S_{74}[\mathrm{Jy}]$ & $23.93 \pm 2.39$ & $38.63 \pm 3.86$ & $19.68 \pm 1.97$ & $421.96 \pm 42.20$ & $17.29 \pm 1.73$ & $28.28 \pm 2.83$ & $31.38 \pm 3.1435 .66 \pm 3.57$ \\
\hline
\end{tabular}

densities with the flux densities observed at close frequencies). For 3C41, both the target and the sources in its close neighbourhood have their TGSS flux densities a few percent lower than the values obtained in the 6CVI survey at $151 \mathrm{MHz}$. In the case of 3C123 and PKS 0235-19 the situation is more complex. Their TGSS flux densities (and those of the sources in their vicinity) are a few percent larger than the corresponding flux densities derived from the GaLactic and Extragalactic All-sky MWA survey (GLEAM, Hurley-Walker et al. 2017) at $150.5 \mathrm{MHz}$. Also, the TGSS-RSADR1 catalogue is not fully conclusive here: for $3 \mathrm{C} 123$, it provides a flux density value even lower than the one obtained with the original TGSS ( 271.70 vs. $272.08 \mathrm{Jy}$ ), while in the case of PKS 0235-19 the flux is actually larger and has a value of $24.20 \mathrm{Jy}$ (vs. 24.12 Jy for TGSS). In the case of 3C41 there is no available flux density provided by TGSS-RSADR1 due to its limited range of declination. Nevertheless it can be clearly proven that these small differences in the flux densities at $151 \mathrm{MHz}$ do not significantly affect the results of the modelling and the final statistical tests used to examine the compared models (cf. Sect. 4). In fact, changing these TGSS values to the values given by TGSS-RSADR1 only results in a difference in the third decimal place for the calculated likelihood ratios.

\section{Application of the KDA EXT model to the sample sources and its comparison to the KDA fits.}

\subsection{Fitting procedure}

In the theoretical considerations, the only unknown parameters are the source's physical parameters, such as injection index $\alpha_{\text {inj }}$, age $t$, the jet's power $Q_{\text {jet }}$, and central density $\rho_{0}$. However, the original KDA model only enables the prediction of the time evolution of the source's observational parameters (jet length $D$, radio luminosity $P_{v}$ at a given frequency, the shape of radio spectrum, and the lobe's volume $V$ ) with the previous assumption about the intrinsic dynamics of this source, defined by the physical quantities mentioned above. This implies that it is first necessary to solve the reverse problem of estimating the initial values of $t, \alpha_{\mathrm{inj}}, Q_{\mathrm{jet}}$, and $\rho_{0}$ in order to subsequently fit the proper KDA or KDA EXT model to the observed source parameters. This was performed using the DYNAGE algorithm (Machalski et al. 2007). In this step, the age resulting from the KDA model $\left(t_{\mathrm{KDA}}\right)$ for the low-frequency parts of the spectra of the sample sources was determined. Here the high-frequency flux densities mostly contributing to the high curvatures of the observed source spectra at above $2000 \mathrm{MHz}$ were excluded from the input data. Additionally, the appropriate correction for the changed length of the jets was taken into account in the KDA model, neglecting the input flux densities at these high frequencies results in fitting the younger age of the source and the lower value of the length of its lobes). This has provided the estimation of the approximate values of four model parameters: $\alpha_{\text {inj }}$, the source's dynamical age, $t$, jet power, $Q_{j}$; and the central core density, $\rho_{0}$. The remaining model parameters were also adopted as in Kuligowska (2017), for example, $\Gamma_{\mathrm{c}}=\Gamma_{\mathrm{a}}=$ $\Gamma_{\mathrm{B}}=5 / 3$ for the "cold" equation of state, and $\beta=1.5$.

Determining the so-called "best age solution" for the initial KDA fit demanded testing some model parameters: here it was assumed that the source's age, $t$, may vary from $10^{4}$ up to $10^{8} \mathrm{yr}$, while the $\alpha_{\text {inj }}$ is from the rather wide range of 0.3 up to 1.0 , and is fitted with the accuracy of 0.01 . In the first (fitting the KDA values) and the second (determining $t_{\mathrm{br}}<t$ ) steps, the best age solutions were fitted with an accuracy of 0.01 million years (Myr). It should be noted that $t_{\mathrm{br}}<t$ solutions are unambiguous: there are clear and single minima of the obtained $\chi^{2}$ values in the function of tested $t_{\mathrm{br}}<t$ values.

In the second step, the values of source power $P_{v_{\mathrm{em}}}$ given by Eq. (6) at the given frequencies for a number of arbitrarily chosen values for the jet switch-off time $t_{\mathrm{br}}$, fulfilling the $t_{\mathrm{br}}<t$ condition, were calculated. Then, the best fit of these model results was determined using the least-squares method by minimizing the expression:

$\chi_{\text {red }}^{2}=\frac{1}{n-r-1} \sum_{n}\left(\frac{S_{v_{0}}-S_{\mathrm{MOD}}}{\Delta S_{v_{0}}}\right)^{2}$,

where $n$ is the number of the compared values (flux densities), $r$ is the number of the model's free parameters, $S_{\nu_{0}}$ and $\Delta S_{\nu_{0}}$ are flux densities, and $S_{\text {MOD }}$ are the model flux densities recalculated from the model values of the emitted power $P_{v_{\mathrm{em}}}$ according to

$S_{\mathrm{MOD}}=P_{v_{e m}}\left(\frac{1+z}{L_{D}^{2}(z)}\right)=P_{v_{0}(1+z)}\left(\frac{1+z}{L_{D}^{2}(z)}\right)$.

The $S_{\text {MOD }}$ flux densities, resulting from the KDA (best age solution) and KDA EXT models, are given in Tables 2-9 along with the values of $\chi^{2}$, determining the goodness of the resulting fits, and the numbers of their free parameters, r. The accuracy of both the fitted models is compared to the observed flux densities representing the real radio spectra of the sample sources. In addition, the results of the likelihood ratio test $(\Lambda)$ were calculated for the compared KDA and KDA EXT best model fits, based on their $\chi^{2}$ distributions and for the assumed level of significance of $10 \%$ (0.01), in order to obtain the reliable statistical tool for the model selection (providing the formal statistical preference of one model over another). The use of this tool is justified here by the fact that the KDA and the KDA EXT models are nested (KDA EXT is a more general case of the KDA, meaning all the parameters of the simpler KDA model also occur in the KDAEXT, and the KDA model may be derived directly from KDAEXT by setting one of its free parameters, $t_{\mathrm{br}}$, to 0$)$. It is worth noting that the KDAEXT has only one more degree of freedom than the KDA (associated with $t_{\mathrm{br}}$ ).

The formal values of the flux density uncertainties resulting from the KDA and KDA EXT models are also provided. These 
uncertainties include both the uncertainties of the calculated radio powers $\left(P_{v}\right)$, and the errors of the redshift values known from the literature. It should be noted, however, that these uncertainties are rather crude, especially due to the lack of reliable redshift uncertainties. They are known only for three of the eight analysed sources (cf. Table 1), and for the remaining ones the rough values of the redshift error are equal to 0.001 (the higher known redshift uncertainty from the examined sources sample) have been adopted. As a result, the final flux density errors may be overvalued or underestimated. Such approximate uncertainties, however, are only supposed to provide an overall view on the capabilities and limitations of the KDA and KDA EXT models (cf. Sect. 5).

\subsection{Fitting results}

Table 10 presents the values of the model free parameters and derived physical parameters of the sample sources resulting from the best fit of the KDA and KDAEXT models. These derived parameters are the cocoon pressure, $p_{\mathrm{c}}$; the total emitted energy, $U_{\mathrm{c}}$; the strength of the magnetic field, $B_{\mathrm{eq}}$; and the radial expansion speed of the cocoon's head, $v_{\mathrm{h}}$. It is worth noting that their values are calculated for $t>t_{\mathrm{br}}$ (after the switchoff), analogous to the formulae given by Kuligowska (2017). Namely, $v_{\mathrm{h}}(t)$ and $p_{\mathrm{c}}$ for $t>t_{\mathrm{br}}$ are calculated with Eqs. (11) and (12) of Kuligowska (2017), respectively, and $U_{\mathrm{c}}$ is given by the formula $U_{\mathrm{c}}(t)=u_{\mathrm{c}}(t) V_{\mathrm{c}}(t)=p_{\mathrm{c}}(t) V_{\mathrm{c}}(t) /\left(\Gamma_{\mathrm{c}}-1\right)$. A compilation of the results obtained for the individual sources from the sample is presented below. In this paper the given errors have been enlarged due to their initial incompleteness, presumably resulting from not taking into account all of the error components. Following the typical percentage error values estimated, for example, for VLSS flux densities, it was arbitrarily assumed that the actual error values can be up to $10 \%$ of the given flux density for the observational frequency range $20-750 \mathrm{MHz}$, and equal to about $5 \%$ for the higher frequencies. The $\chi^{2}$ values in Tables 2-9 are derived with the errors defined in the above way.

\subsection{1. $3 \mathrm{C} 006.1$}

3C006.1 is an example of classical FR II-type radio source with long lobes and distinct hot spots. It lies at relatively high redshift $(z=0.84)$ and was previously studied by Neff et al. (1995). It is worth noting that the radio core of this galaxy was not detected in the VLA maps at $6 \mathrm{~cm}$, and the authors argue that its nucleus may have changed its spatial orientation. This guess is based on radio morphology of the source: a faint inner lobe structure is visible on VLA radio maps at $20 \mathrm{~cm}$.

The radio spectrum coverage for this source extends from $74 \mathrm{MHz}$ up to $21.7 \mathrm{GHz}$. Such a broad spectrum usually results in reliable KDAEXT or KDA model fits to the observed flux densities. However, in this case the strong bend typical for old FR II-type sources is visible only at extremely high frequencies (starting from $10.7 \mathrm{GHz}$ ), and both fitted models are rather poor, especially in comparison with other objects from the sample. This source also has a very flat low-frequency part of the spectrum (spectral index between 74 and $178 \mathrm{MHz}$ equal to 0.45 ), making it difficult to determine the initial parameters of the models. As a result, spectra obtained from the predicted flux densities $S_{\text {MOD }}$ (KDA) and $S_{\text {MOD }}$ (KDA EXT) (Fig. 1) depart from both low- and high-frequency data points, though the KDA EXT visually fits better to the observed flux densities and the provided result for the likelihood ratio test clearly indicates a preference for the KDA EXT over the KDA model.
Table 2. Model flux densities $S_{\mathrm{MOD}}$ from the KDA and KDA EXT fitting for 3C006.1 and their goodness of fit to the observed data $S_{v_{0}}$.

\begin{tabular}{|c|c|c|c|}
\hline$v_{0}[\mathrm{MHz}]$ & $S_{v_{0}} \pm \Delta[\mathrm{Jy}]$ & $\begin{array}{l}S_{\mathrm{MOD}}[\mathrm{Jy}] \\
\text { KDA EXT }\end{array}$ & $\begin{array}{r}S_{\mathrm{MOD}}[\mathrm{Jy}] \\
\mathrm{KDA}\end{array}$ \\
\hline 74 & $23.93 \pm 2.39$ & $32.32 \pm 3.24$ & $31.44 \pm 3.24$ \\
\hline 150 & $18.27 \pm 1.83$ & $19.21 \pm 1.93$ & $18.48 \pm 1.93$ \\
\hline 178 & $15.13 \pm 1.51$ & $16.84 \pm 1.69$ & $16.36 \pm 1.69$ \\
\hline 232 & $13.45 \pm 1.34$ & $13.69 \pm 1.37$ & $13.23 \pm 1.37$ \\
\hline 325 & $9.96 \pm 1.00$ & $10.46 \pm 1.05$ & $9.98 \pm 1.05$ \\
\hline 1400 & $3.65 \pm 0.36$ & $3.06 \pm 0.30$ & $3.00 \pm 0.30$ \\
\hline 2260 & $2.20 \pm 0.22$ & $2.01 \pm 0.20$ & $1.97 \pm 0.20$ \\
\hline 2695 & $1.87 \pm 0.19$ & $1.73 \pm 0.17$ & $1.68 \pm 0.17$ \\
\hline 3900 & $1.42 \pm 0.14$ & $1.25 \pm 0.13$ & $1.20 \pm 0.13$ \\
\hline 4800 & $1.10 \pm 0.06$ & $1.03 \pm 0.10$ & $1.00 \pm 0.10$ \\
\hline 4900 & $1.07 \pm 0.05$ & $1.02 \pm 0.10$ & $0.98 \pm 0.10$ \\
\hline 5000 & $1.03 \pm 0.05$ & $0.99 \pm 0.10$ & $0.96 \pm 0.10$ \\
\hline 7700 & $0.64 \pm 0.03$ & $0.66 \pm 0.07$ & $0.65 \pm 0.07$ \\
\hline 8390 & $0.60 \pm 0.03$ & $0.61 \pm 0.06$ & $0.60 \pm 0.06$ \\
\hline 10700 & $0.46 \pm 0.02$ & $0.47 \pm 0.05$ & $0.48 \pm 0.05$ \\
\hline 11200 & $0.42 \pm 0.02$ & $0.45 \pm 0.05$ & $0.46 \pm 0.05$ \\
\hline 14900 & $0.27 \pm 0.01$ & $0.31 \pm 0.03$ & $0.35 \pm 0.03$ \\
\hline 21740 & $0.14 \pm 0.01$ & $0.18 \pm 0.02$ & $0.25 \pm 0.02$ \\
\hline$r$ & & 4 & 3 \\
\hline$\chi^{2}$ & $\Lambda=0.03$ & $\begin{array}{r}4.97 \\
(p=0.1)\end{array}$ & 21.39 \\
\hline
\end{tabular}

Table 3. Model flux densities $S_{\mathrm{MOD}}$ from the KDA and KDAEXT fitting for 3C32 and their goodness of fit to the observed data $S_{v_{0}}$.

\begin{tabular}{rrrr}
\hline \hline$v_{0}[\mathrm{MHz}]$ & $S_{v_{0}} \pm \Delta[\mathrm{Jy}]$ & $\begin{array}{r}S_{\mathrm{MOD}}[\mathrm{Jy}] \\
\text { KDAEXT }\end{array}$ & $\begin{array}{r}S_{\mathrm{MOD}}[\mathrm{Jy}] \\
\text { KDA }\end{array}$ \\
\hline 74 & $38.63 \pm 3.86$ & $44.37 \pm 4.47$ & $51.89 \pm 5.23$ \\
150 & $27.82 \pm 2.78$ & $27.30 \pm 2.75$ & $27.82 \pm 2.80$ \\
160 & $23.60 \pm 2.36$ & $25.59 \pm 2.58$ & $26.43 \pm 2.66$ \\
365 & $14.23 \pm 1.42$ & $13.40 \pm 1.35$ & $12.36 \pm 1.25$ \\
750 & $8.59 \pm 0.86$ & $7.28 \pm 0.73$ & $6.20 \pm 0.62$ \\
1400 & $4.20 \pm 0.21$ & $4.14 \pm 0.42$ & $3.36 \pm 0.34$ \\
2695 & $2.11 \pm 0.11$ & $2.19 \pm 0.22$ & $1.74 \pm 0.18$ \\
2700 & $2.25 \pm 0.11$ & $2.19 \pm 0.22$ & $1.74 \pm 0.18$ \\
4850 & $1.23 \pm 0.06$ & $1.18 \pm 0.12$ & $0.96 \pm 0.10$ \\
5009 & $1.17 \pm 0.06$ & $1.14 \pm 0.11$ & $0.93 \pm 0.09$ \\
10695 & $0.31 \pm 0.02$ & $0.43 \pm 0.05$ & $0.43 \pm 0.04$ \\
\hline$r$ & & 4 & 3 \\
$\chi^{2}$ & & 9.80 & 23.37 \\
& $\Lambda=0.002$ & $(p=0.1)$ &
\end{tabular}

\subsection{2. $3 \mathrm{C} 32$}

The radio source $3 \mathrm{C} 32$ is the largest source in the examined sample, with its lobes totalling $385.7 \mathrm{kpc}$ in length. Its central AGN have been classified as Seyfert type 2 galaxy by Véron-Cetty \& Véron (2006). The spectra resulting from the predicted flux densities $S_{\text {MOD }}(\mathrm{KDA})$ and $S_{\text {MOD }}$ (KDA EXT) are shown in Fig. 2. In this case, the preference of the KDA EXT model over KDA model is evident, with the $\Lambda$ value of 0.002 for a level of significance of $10 \%$. However, the goodness of the fit to data points $\left(\chi^{2}\right.$ is not high and some observational data points are very far away from the trend line (compare Table 3)). 
Table 4. Model flux densities $S_{\mathrm{MOD}}$ from the KDA and KDAEXT fitting for 3C41 and their goodness of fit to the observed data $S_{v_{0}}$.

\begin{tabular}{rrrr}
\hline \hline$v_{0}[\mathrm{MHz}]$ & $S_{v_{0}} \pm \Delta[\mathrm{Jy}]$ & $\begin{array}{r}S_{\text {MOD }}[\mathrm{Jy}] \\
\text { KDA EXT }\end{array}$ & $\begin{array}{r}S_{\text {MOD }}[\mathrm{Jy}] \\
\text { KDA }\end{array}$ \\
\hline 74 & $19.68 \pm 1.97$ & $21.47 \pm 2.98$ & $29.71 \pm 2.16$ \\
112 & $15.51 \pm 1.55$ & $17.33 \pm 2.25$ & $22.33 \pm 1.74$ \\
150 & $12.20 \pm 1.22$ & $14.75 \pm 1.82$ & $18.11 \pm 1.48$ \\
160 & $14.20 \pm 1.42$ & $14.32 \pm 1.74$ & $17.29 \pm 1.44$ \\
232 & $11.21 \pm 1.12$ & $11.69 \pm 1.32$ & $13.15 \pm 1.18$ \\
325 & $9.39 \pm 0.94$ & $9.63 \pm 1.03$ & $10.21 \pm 0.98$ \\
365 & $9.40 \pm 0.94$ & $9.01 \pm 0.94$ & $9.33 \pm 0.91$ \\
1400 & $3.71 \pm 0.19$ & $3.82 \pm 0.32$ & $3.22 \pm 0.39$ \\
1410 & $3.65 \pm 0.18$ & $3.80 \pm 0.32$ & $3.21 \pm 0.38$ \\
1670 & $3.43 \pm 0.17$ & $3.37 \pm 0.28$ & $2.79 \pm 0.34$ \\
2695 & $2.26 \pm 0.11$ & $2.36 \pm 0.19$ & $1.88 \pm 0.24$ \\
2700 & $2.30 \pm 0.12$ & $2.35 \pm 0.19$ & $1.88 \pm 0.24$ \\
4850 & $1.47 \pm 0.07$ & $1.47 \pm 0.12$ & $1.16 \pm 0.15$ \\
5000 & $1.46 \pm 0.07$ & $1.44 \pm 0.11$ & $1.12 \pm 0.14$ \\
10695 & $0.76 \pm 0.04$ & $0.73 \pm 0.06$ & $0.59 \pm 0.07$ \\
14900 & $0.51 \pm 0.03$ & $0.53 \pm 0.05$ & $0.45 \pm 0.05$ \\
31400 & $0.21 \pm 0.01$ & $0.24 \pm 0.02$ & $0.24 \pm 0.02$ \\
\hline$r$ & & 4 & 3 \\
$\chi^{2}$ & & 1.62 & 15.37 \\
& $\Lambda=0.11$ & $(p=0.1)$ & \\
\hline & & &
\end{tabular}

\subsection{3. $3 \mathrm{C} 41$}

Radio source 3C41 shows classic extended FR II-type morphology and lies at relatively high $z$. It is worth noting that its KDAEXT model fit, presented in Fig. 3, seems very reliable, with a resulting $\chi^{2}$ value almost ten times less than the corresponding value for KDA model. The likelihood ratio test, however, suggests that there is no significant preference of the KDA EXT model over KDA, or at least the results of both models are statistically comparable, with $\Lambda$ slightly higher than the assumed significance level. The spectra calculated from both fitted models are also given in Table 4. Interestingly, in the case of $3 \mathrm{C} 41$, the flat low-frequency part of the KDA EXT radio spectrum visually fits the observed data well, regardless of the poor statistical $\Lambda$ result.

\subsection{4. $3 \mathrm{C} 123$}

This next radio source from the $3 \mathrm{C}$ catalogue is classified as a FR II-type galaxy with extended tails of both lobe components (Fanaroff \& Riley 1974). It is also characterised by a relatively low redshift $(z=0.218$, Spinrad et al. 1985) and short lobes (compare Table 1). 3C123 also shows exceptionally high radio luminosity and non-typical radio structure Riley \& Pooley (1978). Its hot spots along with the general spectral analysis were studied in detail by Looney \& Hardcastle (2000) with the highest resolution to date $\left(0.6^{\prime \prime}\right)$. The authors concluded that $3 \mathrm{C} 123$ may have two pairs of (primary and secondary) hot spots, and there is no clear evidence for a continuing outflow between these hot spots, suggesting that the source may be undergoing recurrent activity. If this source is actually restarting, one could argue it would be more effective to use the model of Brocksopp et al. (2011) which also introduces a free parameter related to the age at which energy supply to the jet stops, $t_{\mathrm{j}}$. Nevertheless, 3C123 meets the strict selection criteria described in Sect. 3.2 and here it is simply assumed that the application of the KDA EXT model to the case of this source is also both justified and interesting.
Table 5. Model flux densities $S_{\text {MOD }}$ from the KDA and KDAEXT fitting for $3 \mathrm{C} 123$ and their goodness of fit to the observed data $S_{v_{0}}$.

\begin{tabular}{rrrr}
\hline \hline$v_{0}[\mathrm{MHz}]$ & $S_{v_{0}} \pm[\mathrm{Jy}]$ & $\begin{array}{r}S_{\text {MOD }}[\mathrm{Jy}] \\
\text { KDAEXT }\end{array}$ & $\begin{array}{r}S_{\text {MOD }}[\mathrm{Jy}] \\
\text { KDA }\end{array}$ \\
\hline 26 & $840.10 \pm 84.00$ & $981.23 \pm 98.38$ & $1020.40 \pm 102.31$ \\
38 & $628.26 \pm 62.83$ & $882.61 \pm 88.46$ & $913.63 \pm 91.61$ \\
74 & $421.96 \pm 42.20$ & $691.46 \pm 69.33$ & $707.57 \pm 70.95$ \\
80 & $342.00 \pm 34.20$ & $443.37 \pm 44.46$ & $446.45 \pm 44.76$ \\
150 & $272.08 \pm 27.21$ & $274.26 \pm 27.52$ & $271.14 \pm 27.12$ \\
160 & $226.60 \pm 22.66$ & $256.90 \pm 25.78$ & $255.13 \pm 25.58$ \\
178 & $208.25 \pm 20.83$ & $237.56 \pm 23.82$ & $235.92 \pm 23.66$ \\
318 & $135.45 \pm 13.55$ & $153.38 \pm 15.37$ & $150.93 \pm 15.13$ \\
325 & $151.07 \pm 15.11$ & $150.58 \pm 15.09$ & $148.17 \pm 14.86$ \\
327 & $148.88 \pm 14.89$ & $149.89 \pm 15.01$ & $147.49 \pm 14.79$ \\
408 & $122.34 \pm 12.23$ & $126.12 \pm 12.65$ & $123.53 \pm 12.39$ \\
750 & $80.10 \pm 8.01$ & $76.87 \pm 7.71$ & $74.95 \pm 7.51$ \\
1400 & $49.73 \pm 2.49$ & $45.16 \pm 4.53$ & $43.63 \pm 4.37$ \\
2695 & $27.50 \pm 1.38$ & $25.16 \pm 2.052$ & $24.14 \pm 2.37$ \\
2700 & $23.35 \pm 1.17$ & $25.16 \pm 2.052$ & $24.09 \pm 2.42$ \\
4850 & $16.03 \pm 0.80$ & $14.65 \pm 1.48$ & $13.89 \pm 1.40$ \\
8420 & $10.15 \pm 0.51$ & $8.67 \pm 0.87$ & $8.12 \pm 0.81$ \\
10500 & $7.90 \pm 0.40$ & $7.01 \pm 0.70$ & $6.53 \pm 0.65$ \\
10695 & $8.18 \pm 0.41$ & $6.88 \pm 0.69$ & $6.41 \pm 0.64$ \\
14900 & $5.30 \pm 0.27$ & $4.99 \pm 0.50$ & $4.60 \pm 0.46$ \\
15300 & $5.41 \pm 0.27$ & $4.85 \pm 0.49$ & $4.47 \pm 0.45$ \\
22000 & $3.45 \pm 0.17$ & $3.39 \pm 0.34$ & $3.10 \pm 0.31$ \\
\hline$r$ & & 4 & 3 \\
$\chi 2$ & & 2.96 & 9.72 \\
& $\Lambda=0.72$ & $(p=0.1)$ & \\
\hline & & &
\end{tabular}

The radio spectrum of this source is very broad thanks to its high radio brightness. Surprisingly, analysis shows that there is no major difference between the spectra resulting from the predicted flux densities for KDA EXT and KDA models (Fig. 4), though the KDAEXT fit is slightly more consistent with observations. There is also no statistical preference of the KDAEXT model, according to the obtained $\Lambda$ value for the adopted level of significance (Table 5).

\subsection{5. $3 \mathrm{C} 247$}

3C247 is another example of a FR II-type radio galaxy at rather high redshift. It was previously studied by Laing et al. (1983) who suggested that its parent galaxy coincides with a weak radio core. Its Multi Element Radio Linked Interferometer Network (MERLIN) radio map (Chidi et al. 1994) shows rather thin and elongated lobes with bright hot spots. The spectra resulting from the predicted flux densities for KDA EXT and KDA models are presented in Fig. 5, and the exact flux density values, along with the goodness of the fit to the data and the likelihood ratio analysis for the models, are listed in Table 6. Although visually the KDAEXT model predicts the observed spectra of 3C247 with better accuracy, the resulting $\Lambda$ value does not confirm a preference for this model over the KDA model.

\subsection{6. $3 \mathrm{C} 325$}

With $z=1.135,3 \mathrm{C} 325$ is the most redshifted galaxy in the sample. It is characterised by a highly curved radio spectrum and a large radio structure with bright lobes. Its classical 
Table 6. Model flux densities $S_{\mathrm{MOD}}$ from the KDA and KDA EXT fitting for 3C247 and their goodness of fit to the observed data $S_{v_{0}}$.

\begin{tabular}{rrrr}
\hline \hline$v_{0}[\mathrm{MHz}]$ & $S_{v_{0}} \pm \Delta[\mathrm{Jy}]$ & $\begin{array}{r}S_{\mathrm{MOD}}[\mathrm{Jy}] \\
\text { KDAEXT }\end{array}$ & $\begin{array}{r}S_{\mathrm{MOD}}[\mathrm{Jy}] \\
\mathrm{KDA}\end{array}$ \\
\hline 74 & $17.29 \pm 1.73$ & $18.64 \pm 1.87$ & $23.74 \pm 2.39$ \\
82 & $16.50 \pm 1.65$ & $17.72 \pm 1.78$ & $22.06 \pm 2.22$ \\
150 & $13.64 \pm 1.36$ & $13.02 \pm 1.31$ & $14.08 \pm 1.42$ \\
232 & $10.31 \pm 1.03$ & $10.22 \pm 1.02$ & $10.11 \pm 1.02$ \\
325 & $8.40 \pm 0.84$ & $8.37 \pm 0.84$ & $7.74 \pm 0.78$ \\
327 & $8.63 \pm 0.86$ & $8.33 \pm 0.84$ & $7.70 \pm 0.77$ \\
365 & $7.92 \pm 0.79$ & $7.77 \pm 0.78$ & $7.04 \pm 0.71$ \\
408 & $7.01 \pm 0.70$ & $7.25 \pm 0.73$ & $6.44 \pm 0.65$ \\
750 & $4.93 \pm 0.49$ & $4.81 \pm 0.48$ & $3.89 \pm 0.39$ \\
1400 & $2.88 \pm 0.14$ & $3.02 \pm 0.30$ & $2.28 \pm 0.23$ \\
2695 & $1.60 \pm 0.08$ & $1.75 \pm 0.18$ & $1.29 \pm 0.13$ \\
2700 & $1.66 \pm 0.08$ & $1.75 \pm 0.18$ & $1.29 \pm 0.13$ \\
4800 & $0.93 \pm 0.05$ & $1.02 \pm 0.10$ & $0.77 \pm 0.08$ \\
4850 & $1.01 \pm 0.05$ & $1.01 \pm 0.10$ & $0.77 \pm 0.08$ \\
4900 & $0.96 \pm 0.05$ & $1.00 \pm 0.10$ & $0.76 \pm 0.08$ \\
5000 & $0.940 \pm 0.05$ & $0.98 \pm 0.09$ & $0.74 \pm 0.07$ \\
10695 & $0.400 \pm 0.02$ & $0.42 \pm 0.04$ & $0.38 \pm 0.04$ \\
14900 & $0.250 \pm 0.01$ & $0.28 \pm 0.03$ & $0.28 \pm 0.03$ \\
\hline$r$ & & 4 & 3 \\
$\chi 2$ & & 1.35 & 11.63 \\
& $\Lambda=0.34$ & $(p=0.1)$ & \\
\hline & & &
\end{tabular}

Table 7. Model flux densities $S_{\text {MOD }}$ from the KDA and KDA EXT fitting for $3 \mathrm{C} 325$ and their goodness of fit to the observed data $S_{v_{0}}$.

\begin{tabular}{rrrr}
\hline \hline$v_{0}[\mathrm{MHz}]$ & $S_{v_{0}} \pm \Delta[\mathrm{Jy}]$ & $\begin{array}{r}S_{\mathrm{MOD}}[\mathrm{Jy}] \\
\text { KDAEXT }\end{array}$ & $\begin{array}{r}S_{\mathrm{MOD}}[\mathrm{Jy}] \\
\mathrm{KDA}\end{array}$ \\
\hline 74 & $28.28 \pm 2.83$ & $37.64 \pm 3.78$ & $39.22 \pm 0.65$ \\
150 & $24.16 \pm 2.42$ & $22.73 \pm 2.28$ & $21.88 \pm 0.56$ \\
178 & $15.85 \pm 1.59$ & $20.07 \pm 2.02$ & $18.86 \pm 0.45$ \\
232 & $14.08 \pm 1.41$ & $16.43 \pm 1.65$ & $15.01 \pm 0.33$ \\
327 & $12.08 \pm 1.21$ & $12.52 \pm 1.26$ & $11.11 \pm 0.16$ \\
750 & $6.38 \pm 0.64$ & $6.23 \pm 0.63$ & $5.25 \pm 0.12$ \\
966 & $5.09 \pm 0.26$ & $4.97 \pm 0.50$ & $4.16 \pm 0.08$ \\
1400 & $3.56 \pm 0.18$ & $3.55 \pm 0.36$ & $2.96 \pm 0.05$ \\
2700 & $1.89 \pm 0.10$ & $1.89 \pm 0.19$ & $1.60 \pm 0.03$ \\
4850 & $0.98 \pm 0.05$ & $1.03 \pm 0.10$ & $0.92 \pm 0.01$ \\
10695 & $0.42 \pm 0.02$ & $0.42 \pm 0.04$ & $0.43 \pm 0.01$ \\
14900 & $0.25 \pm 0.01$ & $0.27 \pm 0.03$ & $0.31 \pm 0.01$ \\
\hline$r$ & & 4 & 3 \\
$\chi^{2}$ & & 3.45 & 10.60 \\
& $\Lambda=0.10$ & $(p=0.1)$ & \\
\hline
\end{tabular}

FR II-type morphology is associated with a parent quasar (Véron-Cetty \& Véron 2006). Preference of the KDA EXT over KDA solution is not evident: the calculated $\Lambda$ value is exactly equal to the assumed significance level for the adopted statistical test. Figure 6 shows that this model fits the high-frequency part of the radio spectrum especially well, while departing from the low flux density data points except for the $150 \mathrm{MHz}$ TGSS flux (see also Table 7).

\subsubsection{C401}

$3 \mathrm{C} 401$ is a rather small $(D=67.5 \mathrm{kpc})$ and low-redshifted $(z=0.201)$ FR II-type radio source previously studied by
Table 8. Model flux densities $S_{\text {MOD }}$ from the KDA and KDAEXT fitting for 3C401 and their goodness of fit to the observed data $S_{v_{0}}$.

\begin{tabular}{rrrr}
\hline \hline$v_{0}[\mathrm{MHz}]$ & $S_{v_{0}} \pm \Delta[\mathrm{Jy}]$ & $\begin{array}{r}S_{\mathrm{MOD}}[\mathrm{Jy}] \\
\text { KDA EXT }\end{array}$ & $\begin{array}{r}S_{\mathrm{MOD}}[\mathrm{Jy}] \\
\text { KDA }\end{array}$ \\
\hline 74 & $31.38 \pm 3.14$ & $42.52 \pm 5.46$ & $54.15 \pm 4.29$ \\
150 & $28.02 \pm 2.80$ & $27.74 \pm 2.65$ & $30.32 \pm 2.61$ \\
178 & $23.55 \pm 2.36$ & $24.52 \pm 2.13$ & $26.28 \pm 2.80$ \\
232 & $22.31 \pm 2.23$ & $20.49 \pm 1.60$ & $21.07 \pm 2.47$ \\
325 & $15.31 \pm 1.53$ & $16.17 \pm 1.45$ & $15.91 \pm 1.63$ \\
365 & $14.93 \pm 1.49$ & $14.85 \pm 0.80$ & $14.41 \pm 1.50$ \\
740 & $8.66 \pm 0.87$ & $8.64 \pm 0.63$ & $7.90 \pm 0.78$ \\
966 & $7.11 \pm 0.71$ & $6.96 \pm 0.46$ & $6.28 \pm 0.70$ \\
1400 & $5.07 \pm 0.25$ & $5.08 \pm 0.26$ & $4.55 \pm 0.51$ \\
2695 & $2.82 \pm 0.14$ & $2.82 \pm 0.15$ & $2.54 \pm 0.28$ \\
4850 & $1.58 \pm 0.08$ & $1.59 \pm 0.15$ & $1.48 \pm 0.16$ \\
4900 & $1.55 \pm 0.08$ & $1.57 \pm 0.14$ & $1.47 \pm 0.16$ \\
5000 & $1.36 \pm 0.07$ & $1.54 \pm 0.14$ & $1.44 \pm 0.15$ \\
8000 & $0.90 \pm 0.05$ & $0.93 \pm 0.09$ & $0.93 \pm 0.09$ \\
10695 & $0.67 \pm 0.03$ & $0.67 \pm 0.07$ & $0.70 \pm 0.07$ \\
10800 & $0.64 \pm 0.03$ & $0.66 \pm 0.07$ & $0.69 \pm 0.07$ \\
14900 & $0.43 \pm 0.02$ & $0.44 \pm 0.05$ & $0.51 \pm 0.04$ \\
\hline$r$ & & 4 & 3 \\
$\chi^{2}$ & & 1.81 & 6.61 \\
& $\Lambda=0.69$ & $(p=0.1)$ &
\end{tabular}

Laing et al. (1983). It coincides with a Seyfert galaxy and is characterised by a radio core of medium brightness equal to $28.54 \pm 0.03 \mathrm{mJy}$ (Hardcastle et al. 1998) and high axial ratio of the lobes. In this case, there is no statistical preference of the KDA EXT model fit over the KDA model, with the $\Lambda$ value being almost seven times larger than the level of significance. The spectra resulting from the predicted flux densities $S_{\text {MOD }}$ (KDA) and $S_{\text {MOD }}$ (KDA EXT) are presented in Fig. 7 and listed in Table 8, along with their corresponding goodness of the fit to $\chi^{2}$ data, which is evidently lower in the case of the KDA EXT fit.

\subsubsection{PKS 0235-19}

This is the only source in the sample not covered by the $3 \mathrm{C}$ catalogue due to its location in the southern hemisphere. Nonetheless, PKS 0235-19 is listed in the Parkes Radio Catalogue (Wright \& Otrupcek 1990). Its nucleus has been classified as a Seyfert 1 galaxy with broad Balmer lines (Tadhunter et al. 1998). The spectra predicted with the KDA and KDAEXT models are shown in Fig. 8. The relevant $\chi^{2}$ and $\Lambda$ values, along with the resulting flux densities calculated for these two models, are presented in Table 9, confirming the preference for the KDA EXT solution.

\section{Discussion}

This work concerns the KDAEXT model describing the dynamics of the FR-II-type radio sources after recent cessation of their jet activity (i.e. having low $\left(t-t_{\mathrm{br}}\right) / t_{\mathrm{br}}$ ratios not exceeding 0.25). KDA EXT is based on the KDA model and assumes self-similarity of the particle flow. Thus one may expect this model not to be applicable in the case of presumably very old radio sources whose jets terminated in the distant past. Theoretical considerations along with some earlier modelling results suggest, rather, that, for so-called radio relics (Murgia et al. 2011) 
Table 9. Model flux densities $S_{\mathrm{MOD}}$ from the KDA and KDA EXT fitting for PKS 0235-19 and their goodness of fit to the observed data $S_{v_{0}}$.

\begin{tabular}{rrrr}
\hline \hline$v_{0}[\mathrm{MHz}]$ & $S_{v_{0}} \pm \Delta[\mathrm{Jy}]$ & $\begin{array}{r}S_{\mathrm{MOD}}[\mathrm{Jy}] \\
\text { KDA EXT }\end{array}$ & $\begin{array}{r}S_{\mathrm{MOD}}[\mathrm{Jy}] \\
\text { KDA }\end{array}$ \\
\hline 74 & $35.66 \pm 3.57$ & $45.45 \pm 4.57$ & $60.33 \pm 6.31$ \\
150 & $24.12 \pm 2.41$ & $28.23 \pm 2.84$ & $32.30 \pm 3.93$ \\
160 & $26.50 \pm 2.65$ & $27.01 \pm 2.73$ & $30.73 \pm 3.76$ \\
365 & $15.60 \pm 1.56$ & $14.54 \pm 1.46$ & $14.37 \pm 2.06$ \\
408 & $13.34 \pm 1.33$ & $13.29 \pm 1.34$ & $12.96 \pm 1.89$ \\
635 & $9.49 \pm 0.95$ & $9.22 \pm 0.93$ & $8.50 \pm 1.34$ \\
1400 & $4.62 \pm 0.23$ & $4.55 \pm 0.46$ & $3.94 \pm 0.70$ \\
2300 & $2.96 \pm 0.15$ & $2.81 \pm 0.28$ & $2.41 \pm 0.46$ \\
2700 & $2.41 \pm 0.12$ & $2.39 \pm 0.24$ & $2.05 \pm 0.40$ \\
3900 & $1.58 \pm 0.08$ & $1.62 \pm 0.16$ & $1.42 \pm 0.29$ \\
4850 & $1.31 \pm 0.07$ & $1.27 \pm 0.13$ & $1.14 \pm 0.24$ \\
10695 & $0.44 \pm 0.02$ & $0.47 \pm 0.05$ & $0.51 \pm 0.12$ \\
11200 & $0.37 \pm 0.02$ & $0.44 \pm 0.04$ & $0.49 \pm 0.11$ \\
\hline$r$ & & 4 & 3 \\
$\chi^{2}$ & & 3.71 & 17.59 \\
& $\Lambda=0.013$ & $(p=0.1)$ & \\
\hline
\end{tabular}

\section{C 006.1}

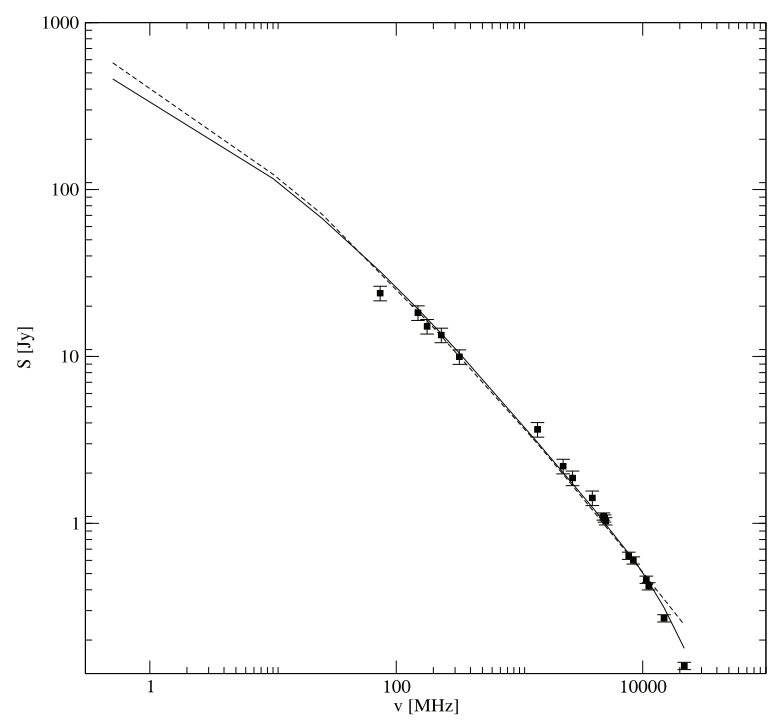

Fig. 1. Best KDA EXT fit (solid line) and KDA fit (dotted line) for radio galaxy 3C006.1. The values of flux density and frequency are presented in logarithmic scale. The observed flux densities and their errors are marked with data points.

in which aging of the radio structure is very evident and the self-similarity is manifestly no longer valid, neither the KDA nor the KDA EXT models can correctly predict their observed radio spectra. Following this supposition, the presented research was focused on those sources showing only modest spectral aging.

This paper is an extended illustration of the general predictions and qualitative analysis of the KDAEXT model, but is restricted due to the very limited sample of the analysed sources. The number of sources showing both the FR II structure and strongly curved radio spectra is small. Future work focussed on proper measurement data for a larger sample of such sources may help greatly in both constraining the parameters of the examined model and evaluation of its reliability.

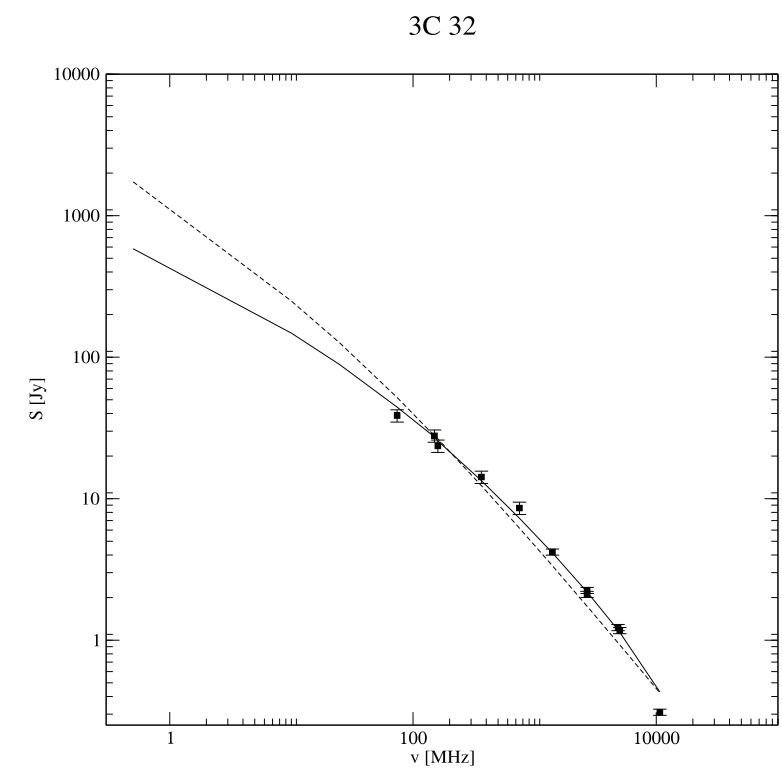

Fig. 2. As in Fig. 1, but for 3C 32 .

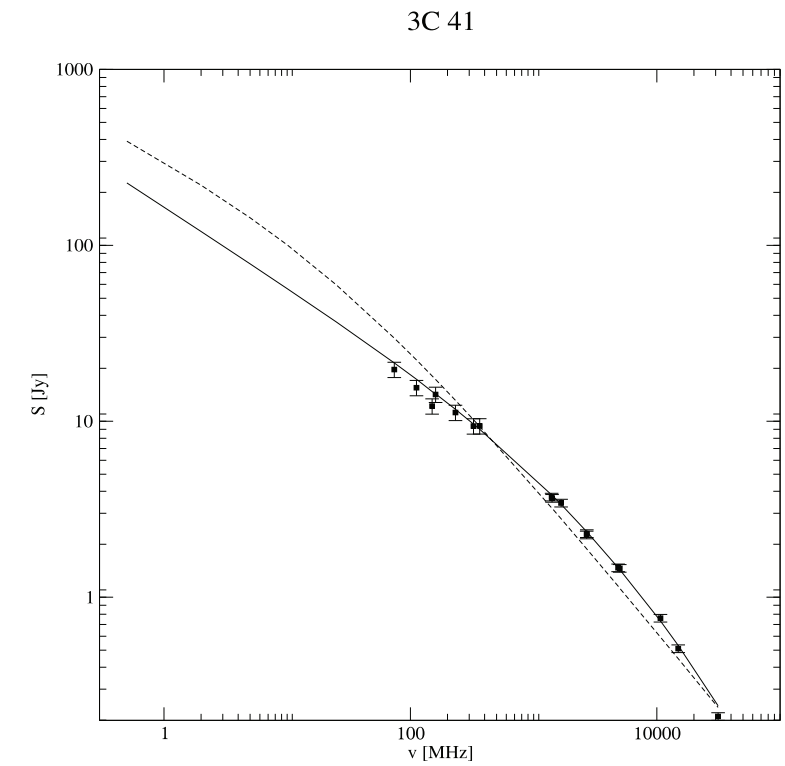

Fig. 3. As in Fig. 1, but for 3C41.

The new results generally confirm those obtained earlier by Kuligowska (2017): in the majority of the analysed cases, the solutions for individual sources summarised in Sect. 4 still show that the KDAEXT model is useful and suitable for studying the sources with strongly bent radio spectra in which central activity terminated relatively recently (or formally: with low $\left.\left(t-t_{\mathrm{br}}\right) / t_{\mathrm{br}}\right)$ ratios). In the context of the presented results (All Figures and Table 10), the most controversial cases are radio sources 3C41 and 3C401. Figure 3 (3C41) shows that in the range of the observed flux densities for this source, the spectrum resulting from the KDA model is initially much steeper than the one obtained with the KDAEXT model. However, the corresponding entries in Table 10 clearly indicate that the $\alpha_{\text {inj }}$ is much lower in the case of the KDA model. This difference is easy to explain if only the wider range of the spectrum (adding the extremely low frequencies ranging from less than $1 \mathrm{MHz}$ to $10 \mathrm{MHz}$ ) is presented. Thus the spectra for both 
Table 10. Physical parameters of the sources from the sample derived from the best KDA fits for the entire available radio spectrum (upper lines) and KDA EXT fits (bottom lines).

\begin{tabular}{lllllllll}
\hline \hline Name & $3 \mathrm{C} 006.1$ & $3 \mathrm{C} 32$ & $3 \mathrm{C} 41$ & $3 \mathrm{C} 123$ & $3 \mathrm{C} 247$ & $3 \mathrm{C} 325$ & $3 \mathrm{C} 401$ & PKS 0235-19 \\
\hline $\mathrm{t}_{\mathrm{KDA}}[\mathrm{Myr}]$ & 19.09 & 45.71 & 9.12 & 2.31 & 26.55 & 31.61 & 0.20 & 25.69 \\
$\mathrm{t}_{\text {KDAEXT }}[\mathrm{Myr}]$ & 19.09 & 38.02 & 2.00 & 1.91 & 9.15 & 17.54 & 6.27 & 25.59 \\
$\mathrm{t}_{\text {br }}[\mathrm{Myr}]$ & 18.43 & 35.91 & 1.73 & 1.82 & 7.31 & 16.60 & 5.71 & 23.22 \\
\hline$\alpha_{\text {inj }}$ & 0.38 & 0.56 & 0.37 & 0.59 & 0.44 & 0.44 & 0.80 & 0.46 \\
& 0.35 & 0.41 & 0.46 & 0.54 & 0.38 & 0.46 & 0.43 & 0.46 \\
$Q_{\text {jet }}[\mathrm{W}]$ & $17.1 \times 10^{38}$ & $3.6 \times 10^{38}$ & $1.9 \times 10^{39}$ & $1.8 \times 10^{39}$ & $4.1 \times 10^{39}$ & $1.5 \times 10^{39}$ & $3.9 \times 10^{39}$ & $8.9 \times 10^{38}$ \\
& $22.7 \times 10^{38}$ & $4.9 \times 10^{38}$ & $3.8 \times 10^{39}$ & $1.5 \times 10^{39}$ & $1.3 \times 10^{39}$ & $8.11 \times 10^{38}$ & $1.01 \times 10^{38}$ & $8.1 \times 10^{38}$ \\
$\rho_{0}\left[\mathrm{~kg} / \mathrm{m}^{3}\right]$ & $4.8 \times 10^{-22}$ & $6.0 \times 10^{-22}$ & $4.8 \times 10^{-22}$ & $1.7 \times 10^{-23}$ & $3.5 \times 10^{-23}$ & $3.5 \times 10^{-22}$ & $1.8 \times 10^{-25}$ & $2.5 \times 10^{-23}$ \\
& $6.0 \times 10^{-22}$ & $5.7 \times 10^{-22}$ & $6.9 \times 10^{-24}$ & $1.9 \times 10^{-23}$ & $7.4 \times 10^{-24}$ & $2.9 \times 10^{-23}$ & $7.7 \times 10^{-23}$ & $2.1 \times 10^{-23}$ \\
$p_{\mathrm{c}}\left[\mathrm{N} / \mathrm{m}^{2}\right]$ & $2.5 \times 10^{-11}$ & $3.9 \times 10^{-12}$ & $5.1 \times 10^{-11}$ & $3.1 \times 10^{-11}$ & $7.1 \times 10^{-12}$ & $1.91 \times 10^{-14}$ & $4.72 \times 10^{-11}$ & $3.01 \times 10^{-12}$ \\
& $3.1 \times 10^{-10}$ & $5.1 \times 10^{-12}$ & $1.6 \times 10^{-11}$ & $4.3 \times 10^{-11}$ & $8.0 \times 10^{-12}$ & $5.11 \times 10^{-12}$ & $2.19 \times 10^{-11}$ & $2.51 \times 10^{-12}$ \\
$U_{\mathrm{c}}[\mathrm{J}]$ & $1.03 \times 10^{54}$ & $5.16 \times 10^{53}$ & $5.38 \times 10^{53}$ & $1.32 \times 10^{53}$ & $3.47 \times 10^{53}$ & $1.50 \times 10^{54}$ & $2.45 \times 10^{52}$ & $7.30 \times 10^{53}$ \\
& $1.36 \times 10^{54}$ & $5.87 \times 10^{52}$ & $2.40 \times 10^{53}$ & $9.22 \times 10^{52}$ & $3.01 \times 10^{53}$ & $5.26 \times 10^{53}$ & $2.0 \times 10^{52}$ & $6.5 \times 10^{53}$ \\
$B_{\text {eq }}[\mathrm{nT}]$ & 6.33 & 2.52 & 9.12 & 7.06 & 3.39 & 5.56 & 8.73 & 2.21 \\
& 7.01 & 2.88 & 5.06 & 8.37 & 3.61 & 2.87 & 5.94 & 2.01 \\
$v_{\mathrm{h}} / c$ & 0.018 & 0.012 & 0.026 & 0.074 & 0.01 & 0.015 & 0.38 & 0.017 \\
& 0.017 & 0.014 & 0.013 & 0.071 & 0.033 & 0.019 & 0.015 & 0.018 \\
\hline
\end{tabular}

Notes. Ages are given in Myr. The accuracy of their numerical determination is of the order of $\pm 0.01 \mathrm{Myr}$.

$3 \mathrm{C} 123$

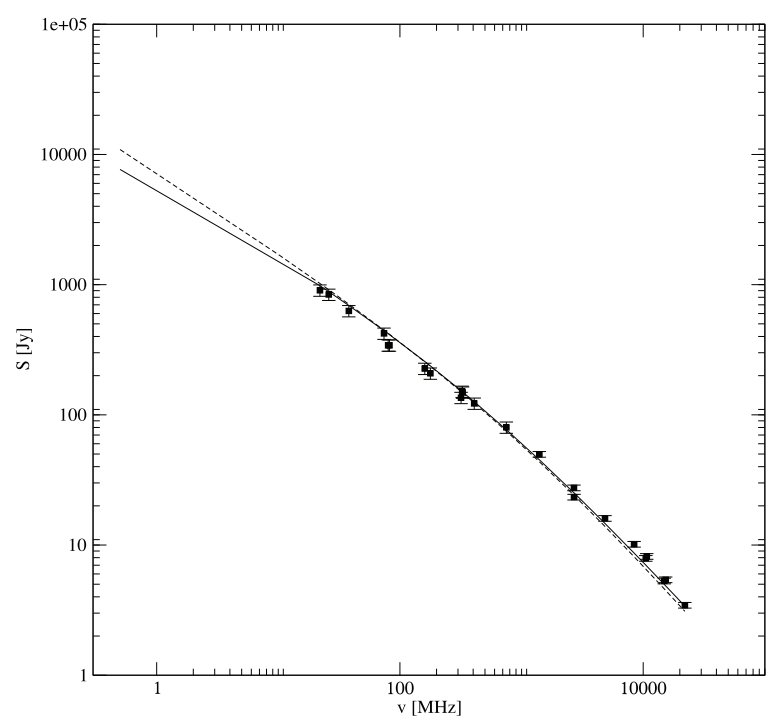

Fig. 4. As in Fig. 1, but for 3C123.

models have been calculated for lower (starting at $0.5 \mathrm{MHz}$ ) frequencies. It should be noted that for the frequencies lower than $10 \mathrm{MHz}$ there is no technical ability to observe radio flux densities with the present-day ground-based telescopes, mostly due to the impact of the ionosphere. The analysis of this low-frequency model spectra confirms that the initial slope is effectively flatter in the case of the KDAEXT model. On the other hand, in the case of 3C401 the resulting KDA and KDAEXT plots (Fig. 7) are not very different in the range of the lower observational frequencies, though the formal measurement uncertainties for both models are rather large in this scope. If, however, the KDA and KDAEXT models for this source are recalculated taking into account the extremely low frequencies lying beyond the observed data points, it becomes clear that the discrimination between $\alpha_{\text {inj }}$ values (also very evident in Table 10)

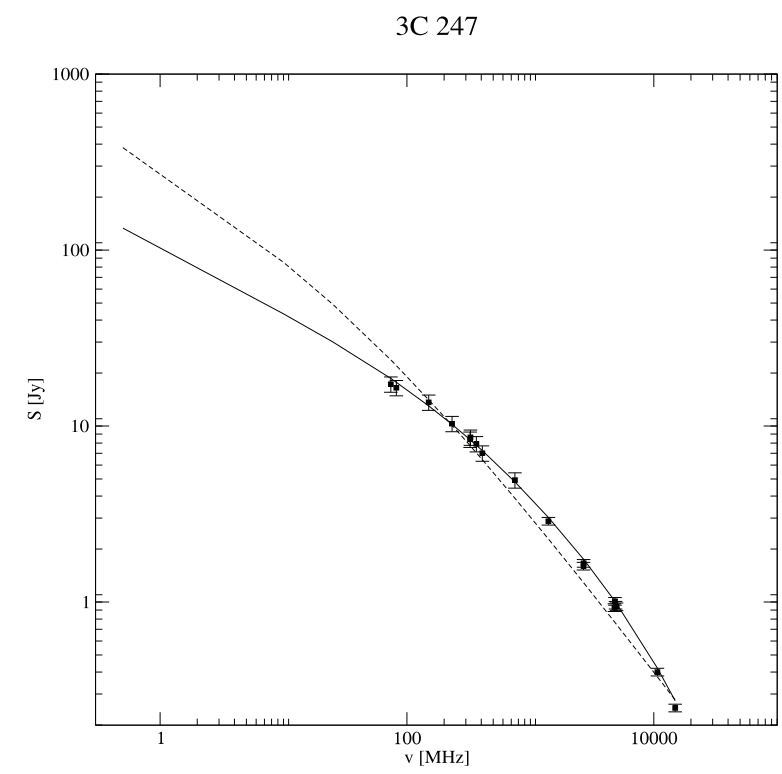

Fig. 5. As in Fig. 1, but for 3C247.

and the overall difference between the two models are actually significant.

The source $3 \mathrm{C} 123$ is also an interesting case. Here, both model (KDA and KDAEXT) fits and their resulting $\chi^{2}$ values are quite similar. Also the applied statistical analysis based on the likelihood ratio test does not confirm the preference of the KDAEXT model. Once again, the solution presented for this source proves that very congruous model-predicted spectral shapes can be obtained starting with not only different models, but also different values of their input parameters (compare Table 10 and the paper of Brocksopp et al. 2011).

Regardless of the apparently curved spectra of the sample sources, an attempt was made to fit their observed spectra with the classical KDA model with high values of the $\alpha_{\text {inj }}$ parameter. Here, conversely, it was assumed that these source are still active and their jets still supply the radio structure. However, it is easy 
$3 \mathrm{C} 325$

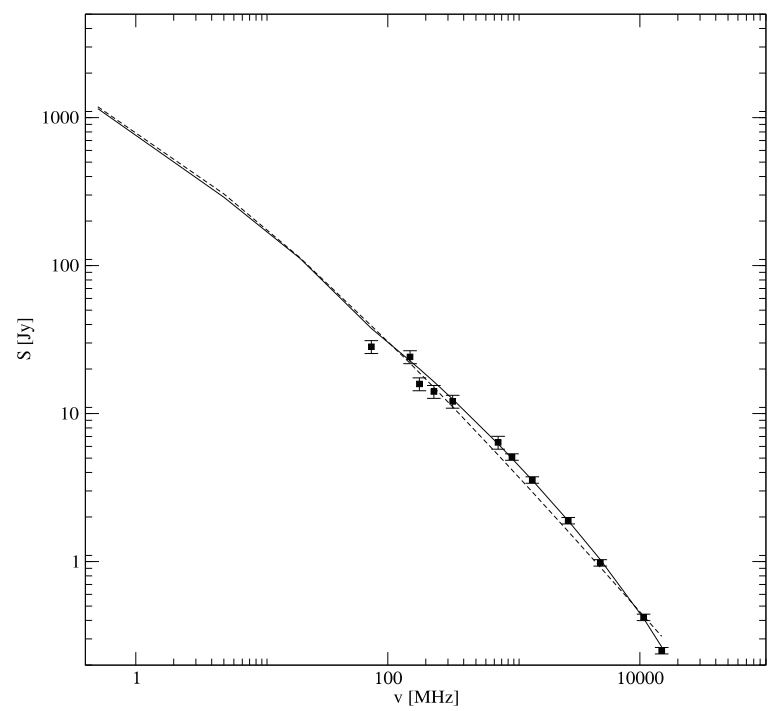

Fig. 6. As in Fig. 1, but for 3C 325 .

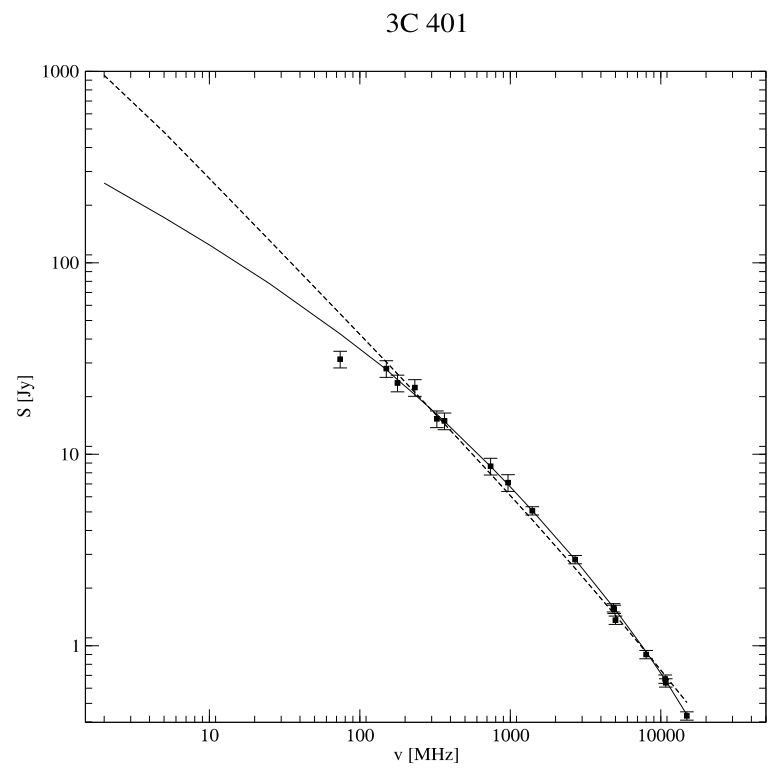

Fig. 7. As in Fig. 1, but for 3C401.

to notice that these resulting fits do not reproduce the observed flux density properly and in the case of 3C401, they result in a very young source age of $0.2 \mathrm{Myr}$.

In addition, the result of the likelihood ratio test clearly indicates that the difference between the KDA and KDAEXT models calculated for this particular source is not statistically significant at the significance level $p=0.10 \%$, despite the large difference in their $\chi^{2}$ values. It may be concluded that there is no statistical preference for the KDA EXT, though the subjective visual plot analysis favours this model.

The results of the KDAEXT modelling presented in this work do not always reproduce the spectra of the sample sources with satisfactory accuracy. There are numerous points departing from the model lines, especially in the low-frequency parts of the predicted spectra. It should be noted that for some sample sources, a good fit of this spectral range was actually achieved, while in other cases a number of low-frequency points lie far from the model (compare 3C325 or 3C401). The same situation
PKS 0235-19

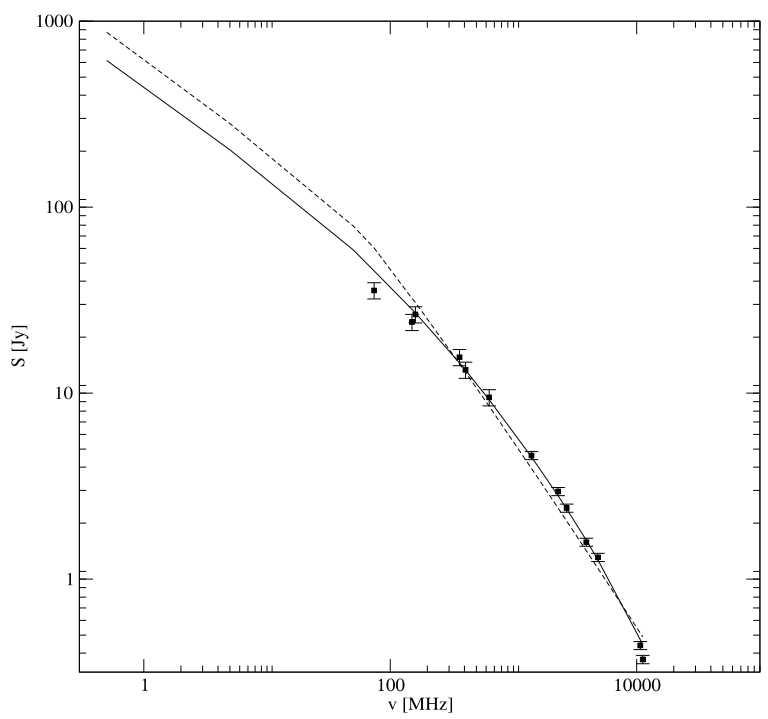

Fig. 8. As in Fig. 1, but for PKS 0235-19.

also applies (to a lesser extent) to the high-frequency parts of the spectra. Except for the underestimated values of the observed flux densities and their errors, the above inconsistency may result from the following factors:

- Variation in the rate of particles transported in the jets (not included in the KDA EXT).

- The source's restarting activity, resulting in another variation in the jet composition.

- General inaccuracy in determining the initial KDA model parameters for the given source for their further use in fitting the KDA EXT model.

- Inaccuracy in estimating the geometrical parameters of the sources such as lobe length or axial ratio, possibly due to the lack of sufficiently detailed radio maps.

The KDAEXT model predicting the evolution of the lobes of FR II-type radio sources after termination of the jet activity, tested here once again on a new sample of galaxies, provides a satisfactory solution for at least four of the eight examined radio sources. Statistically, the KDA EXT model is at least as good, and often better, than the original KDA model, and as such it is a useful tool for fitting and studying the steepened spectra of FR II radio sources.

Acknowledgements. This research was supported by the Polish National Science Centre grant No. 2013/09/B/ST9/00599. The authors express sincere gratitude to the anonymous referee for the useful and essential comments on the manuscript.

\section{References}

Baars, J. W. M., Genzel, R., Pauliny-Toth, I. I. K., \& Witzel, A. 1977, A\&A, 61, 99

Barai, P., \& Wiita, P. J. 2006, MNRAS, 372, 381

Blandford, R. D., \& Rees, M. J. 1974, MNRAS, 169, 395

Blundell, K. M., Rawlings, S., \& Willott, C.J. 1999, AJ, 117, 677

Brocksopp, C., Kaiser, C.R., Schoenmakers, A. P., et al. 2011, MNRAS, 410 484

Chidi, E., Akujor, E., Ludke, I. W. A., et al. 1994, A\&AS, 105, 247

Cohen, A. S., et al. 2007, AJ, 134, 1245

Colla, G., et al. 1970, A\&AS, 1, 281

Edge, D. O., Shakeshaft, J. R., McAdam, W. B., et al. 1959, Mem. R. Astron. Soc., 68, 37

Fanaroff, B. L., \& Riley, J. M. 1974, MNRAS, 167, 31 
A\&A 612, A43 (2018)

Giovannini, G., Feretti, L., Gregorini, L., \& Parma, P. 1988, A\&A, 199, 73

Hardcastle, M. J., Alexander, P., Pooley, G. G., et al. 1998, MNRAS, 296, 445

Heavens, A. F., \& Drury, L. O'C. 1988, MNRAS, 235, 997

Helmboldt, J. F., Kassim, N. E., Cohen, A. S., et al. 2008, ApJS, 174, 313

Hurley-Walker, N. 2017, ArXiv e-prints [arXiv:1703.06635]

Hurley-Walker, N., et al. 2017, MNRAS, 464, 1146

Intema, H. T., Jagannathan, P., Mooley, K. P., \& Frail, D. A. 2017, A\&A, 598, A78

Jaffe, W., \& Perola, G. 1973, A\&A, 26, 423

Kaiser, C. R. 2000, A\&A, 362, 447

Kaiser, C. R., \& Alexander, P. 1997, MNRAS, 286, 215

Kaiser, C. R., \& Cotter, G. 2002, MNRAS, 336, 649

Kaiser, C. R., Dennett-Thorpe, A., \& Alexander, P. 1997, MNRAS, 292, 723

Kaiser, C. R., Schoenmakers, A. P., \& Rottgering, H. J. A. 2000, MNRAS, 315, 381

King, I. R. 1972, ApJ, 174, 123

Kuligowska, E. 2017, A\&A, 598, A93

Laing, R. A., Riley, J. M., \& Longair, M. S. 1983, MNRAS, 204, 151

Looney, L. W., \& Hardcastle, M. J. 2000, ApJ, 534, 172
Machalski, J., Chyży, K. T., Stawarz, Ł., et al. 2007, A\&A, 462, 43

Manolakou, K., \& Kirk, J. G., 2002, A\&A 391, 127

Morganti, R., Killeen, N. E. B., \& Tadhunter, C. N. 1993, MNRAS, 263, 1023

Murgia, M., Parma, P., Mack, K.-H., et al. 2011, A\&A, 526, A148

Neff, S. G., Roberts, L., \& Hutchings, J. B. 1995, ApJS, 99, 349

Pacholczyk, A. G. 1970, Series of Books in Astronomy and Astrophysics (Freeman: San Francisco)

Rawlings, S., Eales, S., \& Lacy, M. 2001, MNRAS, 322, 523

Riley, J. M., \& Pooley, G. G. 1978, MNRAS, 183, 245

Saikia, D. J., Jamrozy, M., Konar, C., et al. 2010, Proc. 25th Texas Symp. Relativistic Astrophysics, Heidelberg, 14

Scheuer, P. A. G. 1974, MNRAS, 166, 513

Shulevski, A., Morganti, R., Barthel, P. D., et al. 2015, A\&A, 583, A89

Spinrad, H., Marr, J., Aguilar, L., \& Djorgovski, S. 1985, PASP, 97, 932

Tadhunter, C. N., Morganti, R., Robinson, A., et al. 1998, MNRAS, 298, 1035

Véron-Cetty, M. P., \& Véron, P. 2006, A\&A, 455, 773

Willot, C., Rawlings, S., \& Blundell, K. M. 2001, MNRAS, 324, 1

Wright, E., \& Otrupcek R., 1990, PASAu, 8, P261 